\title{
Raman spectroscopy vs voltammetry: a voltammetric approach to elucidate different chemicals in a range of pharmaceutical tablets.
}

ALECRIM, M.F., MACÊDO, I.Y.L., RODRIGUES, E.B., MORENO, E.K.G., GHEDINI, P.C., FERNANDEZ, C., DOS SANTOS, W.T.P. and GIL, E.S. 


\title{
Raman Spectroscopy vs Voltammetry: A new voltammetric approach to elucidate different chemicals in a range of pharmaceutical tablets
}

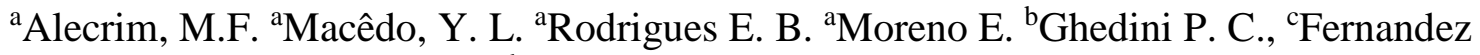 \\ C., ${ }^{\mathrm{d} D o s}$ Santos W. T. P., ${ }^{\mathrm{a}}$ Gil E. S. \\ ${ }^{\text {a }}$ Faculdade de Farmácia, Universidade Federal de Goiás (UFG), Goiânia - GO, Brasil; \\ b Instituto de Ciências Biológicas, Universidade Federal de Goiás (UFG), Goiânia - GO, \\ Brasil; \\ c School of Pharmacy and Life Sciences, Robert Gordon University, Sir Ian Wood \\ Building, AB10 7GJ Aberdeen, United Kingdom; \\ ${ }^{d}$ Departamento de Farmácia, Universidade Federal dos Vales do Jequitinhonha e Mucuri \\ (UFVJM), Diamantina - MG, Brasil.
}

Over the years, Electroanalysis has been widely applied to elucidate redox behavior of novel molecules. The selectivity and low cost are spotlight features in pharmacopeial methods of identification, that can be reached by voltammetric approaches. In this work, differential pulse voltammetric (DPV) profile and the slope of the linear regression obtained from calibration graphs along with the scan study are proposed as new perspective of identification assays. With the proposed methodology we were able to identify the similarities among DPV profile and the slopes obtained for each tablet. In addition, this new technology was successfully employed to identify the following chemicals: Paracetamol (PAR), Promethazine (PMZ), Diclofenac (DIC), Piroxicam (PRX), Indomethacin (IND) and Cyclobenzaprine (CBP) in pharmaceutical assays using Pencil Graphite Electrodes. Furthermore, our new methodology was effectively compared to Raman Spectroscopy for the analysis of the range of chemicals in the pharmaceutical assays.

Keywords: Raman Spectroscopy, Differential Pulse Voltammetry, Pharmaceutical Analysis

\section{Introduction}

Routine laboratory drug analyses for quality control purposes are usually performed through classical, spectrophotometric and chromatographic methods [1]. These techniques often require strenuous pre-preparation of samples, costly equipment, organic solvents and/or time consuming procedures [2, 3]. In this context, electroanalysis has been shown to be an attractive alternative to evaluate of drugs because it presents advantages such as versatility, low cost, simplicity of application, among others. However, these techniques are limited by the low reproducibility, and there is a clear need to evaluate and propose the use of sensors that are easy to clean and that guarantee the recognized advantages of electroanalysis. Hence, the use of graphite pencil electrodes (GPE) has proven to be a useful and efficient and low cost tool for the determination of drugs in pharmaceutical formulations, without many of the drawbacks found in other methods [4-6]. 
On the other hand, the electroanalytical studies have focused the quantitative drug analyses, yielding sensitivity in $\mu \mathrm{mol} \mathrm{\textrm {L } ^ { - 1 }}$ ranges, whereas, there are only a few attempts in identification assays without the use of chemically modified electrodes (CME) [7-10]. Despite the employment of CMEs, voltammetry can display different analytical signals that distinguish species, that might be useful for identification purposes and deserves investigation [11].

Among the instrumental methodologies mostly employed for identification purposes, we have the IV, Raman and NIR spectroscopies [12-15]. The benefits of nondestructive and non-invasive sample analysis provided by Raman spectroscopy, are that reinforces its practical and direct application in pharmaceutical dosage forms without time consuming preparation steps [13, 14]. Meanwhile, though vibrational spectroscopic techniques offer significant singularity for each molecule, the direct use in dosage forms requires chemometrics approaches [13-15]. Indeed, the resulting "multi-peak" spectra are commonly, recognized as a fingerprint of a particular molecule, but this assumption is valid for drugs and excipients [13-15].

Akin, many electroactive compounds can undergo a redox process at the same potential. In turn conforming electrochemical studies under controlled conditions, the relationship between electroactive compounds concentration and anodic peak is directly influenced by the diffusion coefficient and other unique physicochemical properties [16]. Indeed, for all species to reach the electrode surface to undergo redox reactions, there is a dependence of the overpotential that is closely related to the reaction speed of the analyte. The reaction rate, in turn, is influenced by unique molecular characteristics of the compound that act by limiting or favoring the rate of dispersion for electroactive species in the reaction medium [16, 17]. Therefore, when a compound has its mass-transfer controlled by diffusion and this is the limiting step of the reaction on a working electrode, the slope of the calibration curve (peak currents vs different concentrations) is connected to its the diffusion coefficient. Thus, besides the use of calibration curves for compound quantification, the value of the slope between the faradaic signal and the concentration is theoretically singular and even distinguishable, allowing complementary identification of electroactive molecules $[16,17]$.

In the light of the insights, the present work aims to combine the slope obtained from DPV calibration graphs, as an alternative tool of identification. Besides this approach will also provide the identification of different molecules such as: Paracetamol (PAR), Promethazine (PMZ), Diclofenac (DIC), Piroxicam (PRX), Indomethacin (IND) and Cyclobenzaprine (CBP) in pharmaceutical assays by using the slope of the curve that relates the square root with the sweep speed and current. The choice of Graphite Pencil Electrodes have as the foundation on good performance of this material in other studies, highlighting its ease of cleaning, access and low cost. Moreover, the target samples were also identified by Raman spectroscopy, in order, to establish eventual comparisons.

\section{Material and methods}

Materials and samples 
2.0 mm HB graphite rod (Faber-Castel) was purchased from local stationery. Paracetamol (PAR), Promethazine (PMZ), Diclofenac (DIC), Piroxicam (PRX), Indomethacin (IND) and Cyclobenzaprine (CBP) standards (Figure 1) were purchased from Sigma Aldrich (Saint Louis, USA).<smiles>CC(CN1c2ccccc2Sc2ccccc21)N(C)C</smiles>

PMZ<smiles>CC(=O)Nc1ccc(O)cc1</smiles>

PAR<smiles>O=C(O)Cc1ccccc1Nc1c(Cl)cccc1Cl</smiles>

DIC<smiles>COc1ccc2c(c1)c(CC(=O)O)c(C)n2C(=O)c1ccc(Cl)cc1</smiles>

IND<smiles>CN1C(C(=O)Nc2ccccn2)=C(O)c2ccccc2S1(=O)=O</smiles>

PRX

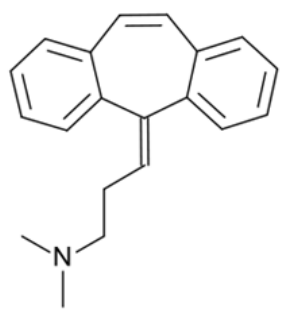

CBP

Figure 1. Chemical structures of PMZ, DIC, PRX, PAR, IND and CBP standards.

The tablets of each selected drug (Table 1) were purchased in a local pharmacy.

TABLE I. Selected target samples description.

\begin{tabular}{lcccc}
\hline Drug & $\begin{array}{c}\text { Dosage } \\
\text { Form }\end{array}$ & $\begin{array}{c}\text { Declared } \\
\text { content } \\
(\mathrm{mg})\end{array}$ & $\begin{array}{c}\text { Average weight } \\
(\mathrm{mg})\end{array}$ & $\begin{array}{c}\text { Therapeutic } \\
\text { Category }\end{array}$ \\
\hline PAR & Tablet & 750 & 836 & mild analgesic \\
DIC & Tablet & 50 & 192 & NSAID* \\
PMZ & Tablet & 25 & 172 & antihistaminic \\
IND & Tablet & 25 & 239 & NSAID* \\
PRX & Capsule & 20 & 309 & NSAID* \\
CBP & Tablet & 10 & 161 & muscle relaxant \\
\hline
\end{tabular}

*Non-steroidal anti-inflammatory drug

Ten tablets or capsules of each target sample were used to estimate the average weight (table 1) and then powdered in a mortar. A suitable amount of each sample was solubilized and centrifuged at $400 \mathrm{rpm}$ for 5 minutes, in order to prepare equivalent molar stock solutions. The identification assays were performed with $32 \mu \mathrm{mol} . \mathrm{L}^{-1}$ assay solutions. The calibration graphs were constructed by using proper aliquots, respecting the linear response of each target sample. All chemicals and solvents were of analytical grade, used without further purification. Stock solutions were prepared prior to the experiments and were diluted in Milli-Q water (conductivity $\leq 0.1 \mu \mathrm{Scm}-1$ ) (Millipore S.A. S., Molsheim, France). 


\subsection{PGE preparation}

The PGE was inserted in a plastic casing, exposing only the circular $2.0 \mathrm{~mm}$ graphite area. The plastic casing was used with the purpose of restricting the contact of the graphite with the solution. After each assay, the pencil was polished in a Jet401 Norton 47F 1200 sandpaper and it was further smoothed in writing paper. The polishing was achieved by drawing 10 full circles of $0.5 \mathrm{~cm}$, whereas smoothing was executed by doing 3 circles of the same size.

\section{Electrochemical assays}

The voltammetric measurements were performed in a potentiostat/galvanostat HAutolab III ${ }^{\circledR}$ integrated with GPES $4.9{ }^{\circledR}$ software, Eco-Chemie, Utrecht, The Netherlands. Measurements were carried out using a three-electrode system in a $2 \mathrm{~mL}$ onecompartment electrochemical cell (Cypress System Inc., USA). PGE ( $\mathrm{d}=2.0 \mathrm{~mm}$ ) were the working electrodes, Pt wire the counter electrode and the Ag/ $\mathrm{AgCl}\left(3 \mathrm{~mol} . \mathrm{L}^{-1} \mathrm{KCl}\right.$ ) reference electrode. The PGE was inserted in a plastic casing, exposing only the circular $2.0 \mathrm{~mm}$ graphite area. The plastic casing was used with the purpose of restricting the contact of the graphite with the solution. A carbon paste electrode (CPE) was prepared as a piston-driven holder containing $70 \%$ of graphite powder and $30 \%$ of $\operatorname{Nujol}{ }^{\circledR}(d=2.0$ $\mathrm{mm}$ ) was also used just for punctual comparisons of robustness. The surface of the carbon paste electrode (CPE) was mechanically renewed after each experiment by extruding approximately $0.5 \mathrm{~mm}$ of carbon paste out of the electrode holder and smoothing it with filter paper. This procedure ensured very reproducible experimental results.

The $\mathrm{pH}$ measurements were carried out with a Quimis $\mathrm{pH}$-meter Q400AS (Quimis ${ }^{\circledR}$, São Paulo, Brazil) with an Ingold combined glass electrode. All experiments were done at room temperature $\left(25 \pm 1^{\circ} \mathrm{C}\right)$ and microvolumes were measured using EP-10 and EP-100 Plus Motorized Microliter Pippettes (Rainin Instrument Co. Inc., Woburn, USA).

The experimental conditions for differential pulse (DP) voltammetry were: pulse amplitude $50 \mathrm{mV}$, pulse width $70 \mathrm{~ms}$ and scan rate $10 \mathrm{mV} \mathrm{s}^{-1}$. For cyclic voltammetry (CV), the scan range from 0 to $1.2 \mathrm{~V}$ at rates of 25, 50,100, 250 and $500 \mathrm{mV} \mathrm{s}^{-1}$ were used. Prior to the DPV assays of each sample, CV scans were recorded in blank buffer solution until a steady state baseline was obtained. This procedure ensured reproducible experimental results. All experiments were performed in triplicate with $\mathrm{pH} 7.00 .1 \mathrm{M}$ phosphate buffer as electrolyte.

Acquisition and Presentation of Voltammetric Data

All the voltammograms presented were background-subtracted and baselinecorrected using the moving average application with a step window of $5 \mathrm{mV}$ included in GPES version 4.9 software. This mathematical treatment improves the visualisation and identification of peaks over the baseline without introducing any artefact. Though the peak intensity can vary slightly ( $<10 \%)$, in comparison to untreated curves, this variation occurs equally for all samples, when the same procedure is applied, being therefore, neglectable. The values for peak current presented in all plots were determined from the original 
untreated voltammograms after subtraction of the baseline. All data was analyzed and treated using the statistical software Origin $9 \circledR$.

Raman assays

A Mira M-1 portable Raman spectrometer (Metrohm, Herisau, Switzerland) equipped with a $785 \mathrm{~nm}$ source and a maximum laser output power of $75 \mathrm{~mW}$ was employed to obtain the spectra. The spectra were recorded in a spectral range of 800 to $1800 \mathrm{~cm}^{-1}$, with average spectral resolution of 12 and $14 \mathrm{~cm}^{-1}$. The measurement was carried out by using a glass vial holder $(12 \times 32 \mathrm{~mm})$ completely filled with c.a. $1 \mathrm{~g}$ of each sample.

Statistical analysis

Statistical analysis was performed using Origin $9 \circledR$. Correlations among slopes obtained from calibration graphs with the standards and dosage forms were made. Both datasets of standard and dosage forms were analyzed individually. A third dataset (standards and dosage forms) was created to established correlation between their parameters. ANOVA and Tukey's test were perfomed for all three datasets. The significance level $(\alpha)$ considered was 0.05 .

\section{Results and discussion}

Raman spectroscopy and differential pulse voltammetry were performed in order to acquire identification data for the following drug standards and their respective tablets: IND, PRC, PRX, PMZ and DIC.

The DPV assays were performed using equal experimental conditions, namely standards of each raw materials, as well as, the powdered tablets, were diluted in order to get $32 \mu \mathrm{mol} . \mathrm{L}^{-1}$ solution, whereas, the electrolyte was $0.1 \mathrm{M}$ phosphate buffer $\mathrm{pH} 7.00$. In turn, the surface area of the GPE was cleaned and polished prior each measurement achieving a rapid and effective analysis. The DPVs of the evaluated drug standards and tablets are shown in Figure 2. 


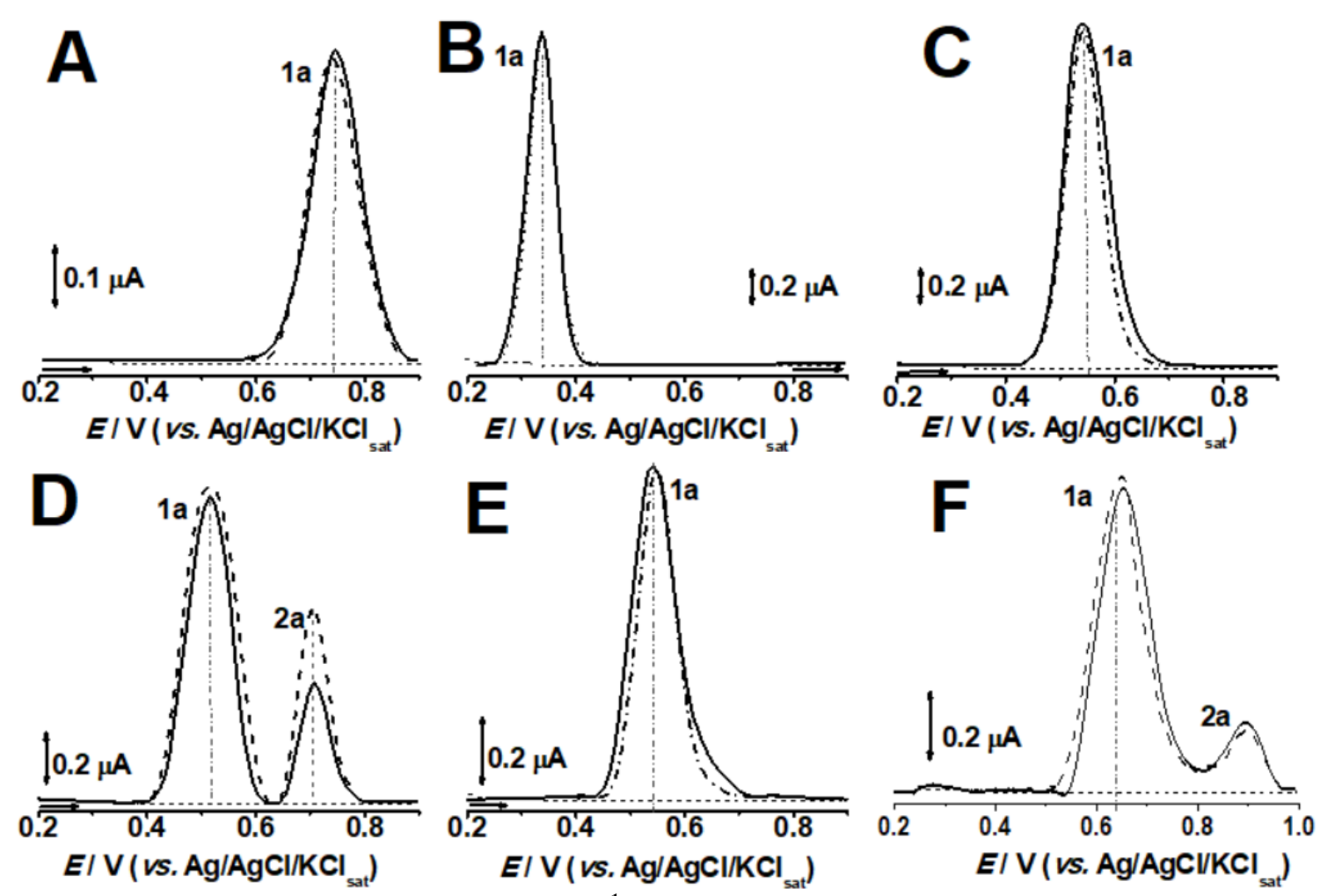

Figure 2. DPVs obtained for $32 \mu \mathrm{mol} . \mathrm{L}^{-1}$ solutions of IND (A), PAR (B), PRX (C), PMZ (D), DIC (E) and CBP (F) for standards (-- ), tablets (-) and 0.1M phosphate buffer, pH $7.0(\ldots . .$.$) . All in 0.1 \mathrm{M}$ phosphate buffer, $\mathrm{pH} 7.0$ at PGE. Amplitude of $50 \mathrm{mV}$, scan rate of $10 \mathrm{mV} / \mathrm{s}$, scan range from 0.2 to $0.9 \mathrm{~V}$.

The DPVs of the target samples herein studied, exhibited peak potentials, at $E_{\text {p1a }}=0.75 \mathrm{~V}$ for IND (Fig. 2A); $E_{\text {p1a }}=0.35 \mathrm{~V}$ for PAR (Fig. 2B); $E_{\text {p1a }}=0.55 \mathrm{~V}$ for PRX (Fig. 2C); $E_{\mathrm{p} 1 \mathrm{a}}=0.55 \mathrm{~V}$ for DIC (Fig. 2E), two anodic peak, 1a and 2a, at $E_{\mathrm{p} 1 \mathrm{a}}=0.52 \mathrm{~V}$ and $E_{\mathrm{p} 2 \mathrm{a}}=0.75 \mathrm{~V}$ for PMZ (Fig. 2D) and for CBP $E_{\mathrm{p} 1 \mathrm{a}}=0.65 \mathrm{~V}$ and $E_{\mathrm{p} 2 \mathrm{a}}=0.9 \mathrm{~V}$ (Fig. 2E).

The voltammetric profile presents some particular distinguishable features, that could allow the tentative identification. For instance, the main electrochemical parameters, peak potential, $E_{\mathrm{p}}$, and peak current, $I_{\mathrm{p}}$, as well as, the number of peaks observed in a particular voltammogram are useful identification patterns. In this sense, except, for DIC (Figure 2C) and PRX (Figure 2E), all target samples exhibited distinguishable differences (Figures 2).

The Raman spectrum of each powdered sample of standards and tablets are shown in Figure 3. 

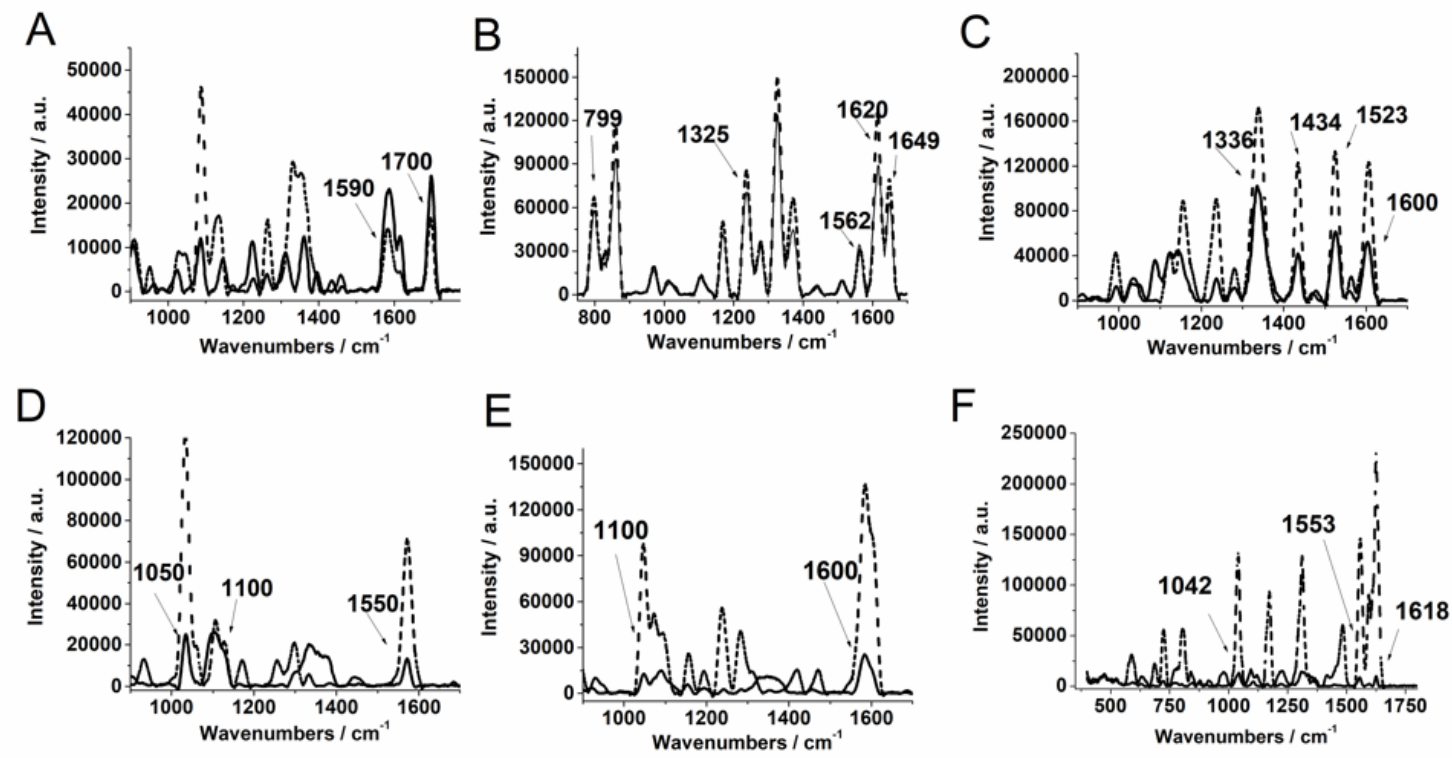

Figure 3. Raman spectrum of the standards (- - ) and tablets (-) of IND (A), PAR (B), PRX (C), PMZ (D), DIC (E) and CBP (F).

The Raman spectrum of each drug showed a unique profile, proving that this technique a viable option [15]. Nevertheless, the Raman spectra of pure standard and its representative tablet shown remarkable differences, especially for higher potency drugs, in which the proportion between active component and excipients is lower. Indeed, the light dispersion of the starch, a common excipient diluent in tablets, occurs between 800-1800 $\mathrm{cm}^{-1}$, and the occlusion of the drugs related peaks is more significant, accordingly to the ratio among declared content and average weight. For instance, the ratio of PMZ, DIC, IND, PRX, and CBP were lower than $15 \%$, producing huge spectral differences. In turn, for PAR $(750 \mathrm{mg} / 835.9 \mathrm{mg})$, the unique case in which the direct identification was feasible, this ratio was almost of $90 \%$. Therefore, the direct identification of a particular drug in dosage forms, in which the interference of excipients is relevant, is possible only with Raman coupled to chemometric tools [14,15, 18,19].

In turn, if the redox behavior of selected target drugs are distinct, since the excipients are mostly non-electroactive species, the prompt identification of drugs may be feasible by DPV even if directly applied to their related dosage forms (Figure 2A, 2B and $2 \mathrm{C}$ or $2 \mathrm{D}, 2 \mathrm{E}$ and $2 \mathrm{~F}$ ). Yet for drugs of similar voltammetric profile (Figure $2 \mathrm{C}$ and $2 \mathrm{E}$ ), this approach would be useful as a complementary identification tool, possessing from nonnegative to indicative status [20].

Since the electroactivity of each specie is centered in different functional groups or molecular sites, the redox process occur in distinguishable ways (Scheme 1S, Supplementary material). Therefore, the extensive electrochemical characterization afford useful insights. For better exploitation in the application of DPV, it is possible that the drugs could be differentiated by the variation of the analysis medium, or by the application of the technique. Figure 4I shows the behavior of successive scans for PRX drugs (Figure 4IA) and DIC (Figure 4IB). In this study the PRX presented an adsorptive profile differently from the DIC, a parameter that may be useful to differentiate drugs, previously indistinguishable, with the use of the technique. While the PRX (Figure 4IA) adsorbs 
strongly on the surface of the electrode, the DIC (Figure 4IB) generates new compounds that are oxidized in a second successive scan. The Figure 4II shows the effect of $\mathrm{pH} 5.00$ and 7.00 to facilitate the differentiation between PMZ (Figure 4IIA) and CBP (Figure 4IIB), since only PMZ exhibits two oxidative processes at $\mathrm{pH}$ 5.00. Thus, $\mathrm{pH}$ variation can be used to identify compounds according to the study design.
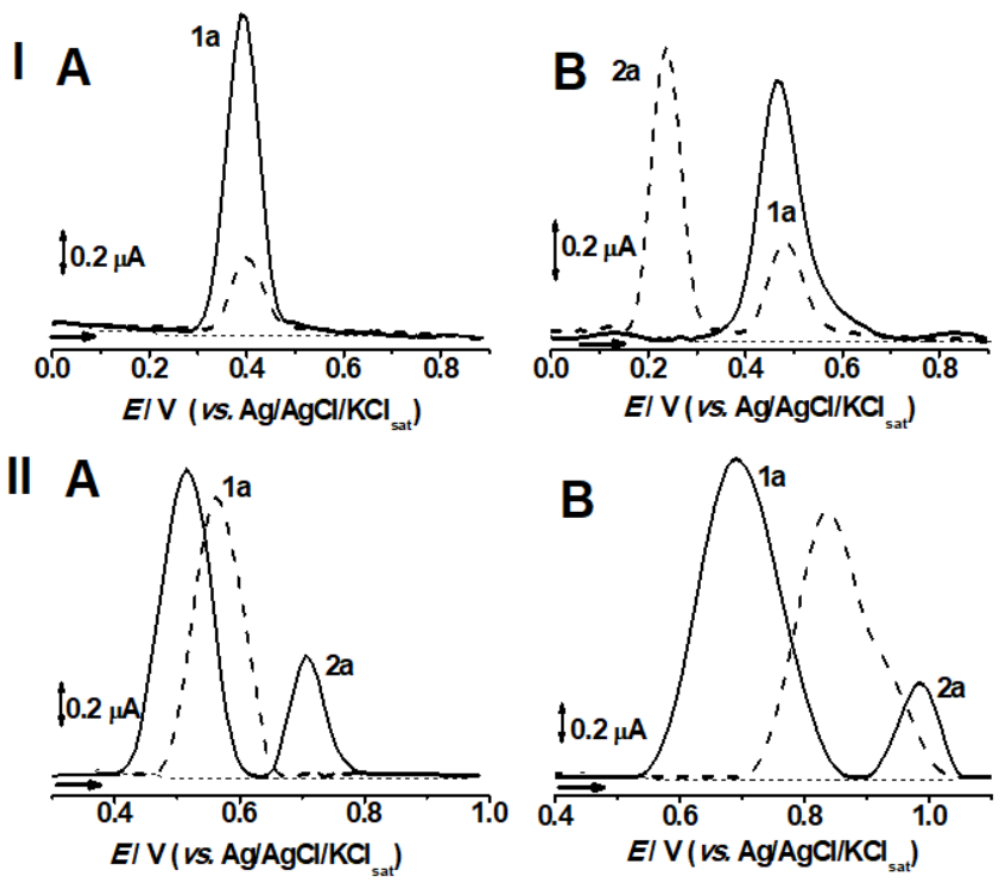

Figure 4. I) DP voltammograms obtained for $32 \mu \mathrm{mol} . \mathrm{L}^{-1}$ solutions of PRX (A) and DIC (B) in pH 7.0 0.1 M PBS, first scan (-), second scan (-- -), and 0.1M phosphate buffer, $\mathrm{pH} 7.0$ (......). II) DP voltammograms obtained for $32 \mu$ mol.L-1 solutions of PMZ (A) and CBP (B) in pH 7.0 0.1 M PBS (-) and in pH 5.0 0.1 M PBS (-- $)$.

Concerning the experimental conditions, some instrumental parameters, i.e. scan range, scan direction and scan rate may have particular impact on the voltammetric profile of each compound. In this context, CV scans were performed at different scan rate for PAR, PMZ, CBP, PRX and DIC. The slopes obtained from $I_{\text {pa }} v s$. square root of scan rate $\left(v^{1 / 2}\right)$ plots presented distinguishable values (Figure 5A).

In the same way, DPV assays at PGE were also carried on the quantification of each drug sample, whereas the corresponding slope obtained from each calibration curves was explored with identification purposes. The resulting graphs (Figure 5B), in which the clear differences endorses the identification process.

The slope was verified on carbon paste electrodes, with the sole purpose of verifying the behavior on distal electrodes. For this, three drugs with closer slopes were chosen when evaluated in PGE (Figure 5C, 5D, 5E). 

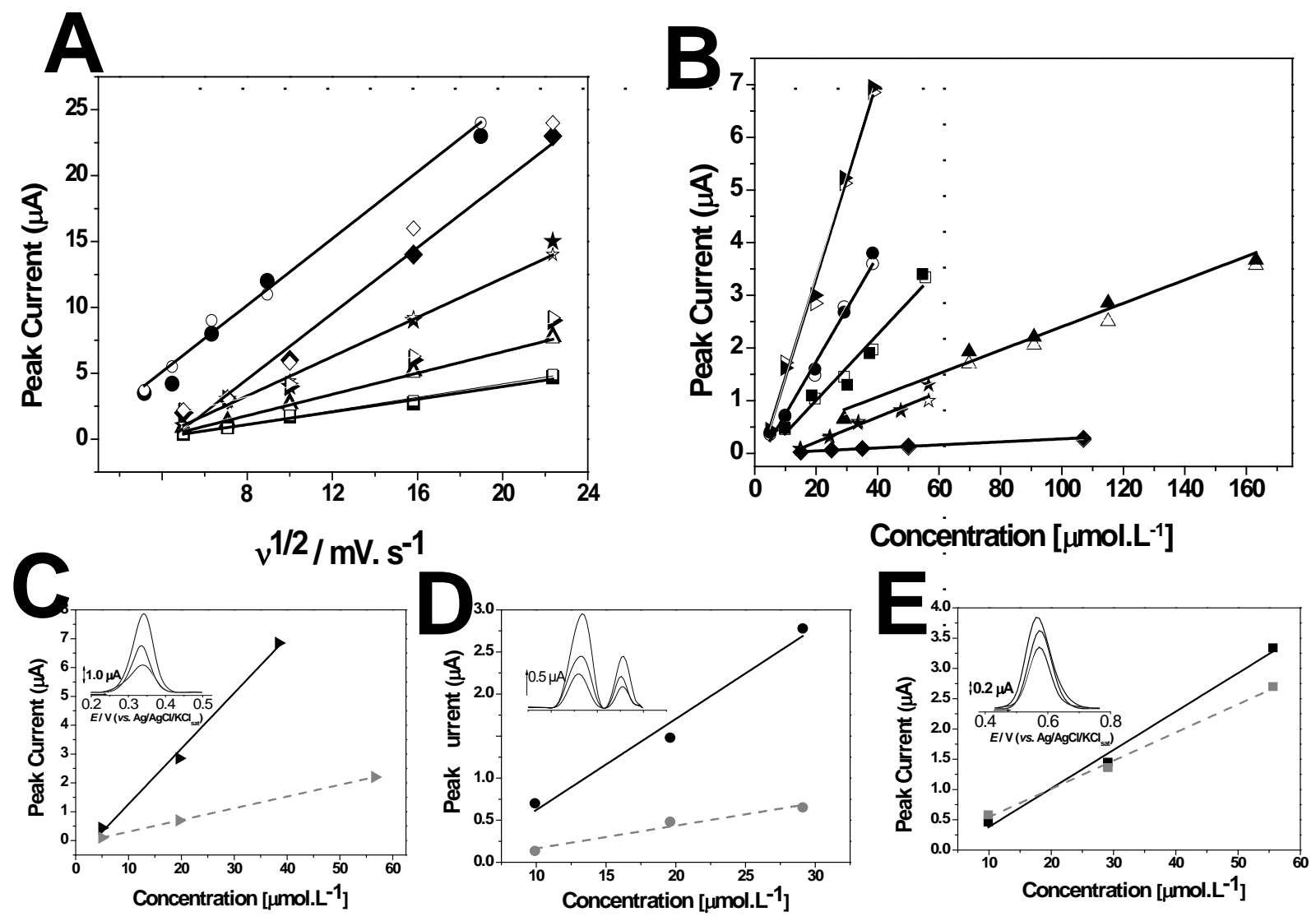

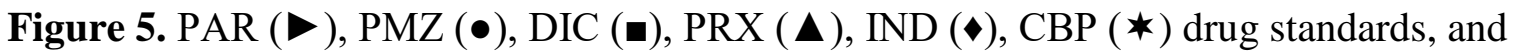
related commercial tablets (blank signs) at PGE in pH 7.0 0.1 M PBS. A) Ip1a vs v1/2 plots obtained at different scan rates (10, 25, 50, 100 and $250 \mathrm{mV.s}-1)$. B) Calibration curves obtained from DPV assays. Assay obtained for three concentration levels at day two of: C) PAR, D) PMZ and E) DIC at the same PGE and in the same experimental conditions described in Figure 4B. The grey (- - ) line show the same experiment at CPE.

The distinct slopes observed for different drugs is in agreement with the crucial role of structural and chemical properties on the electrode kinetics, in which diffusional and electronic limiting factors play different role [16,17].

In order to testify the feasibility of the current proposal, the same procedure was applied for solid dosage forms formulated with the related drugs (Table 1).

When performed at same experimental conditions, the voltammograms and the resulting slopes exhibited identical profiles (Figure 4A and 4B, black and blank signs) for assay solutions prepared from pure standards and from dosage forms (drug plus excipients). Therefore, the differences observed for both slope profiles, evidences that the current intensity is not only consequence of concentration loading of electroactive species, as stated by Faraday Law, but also related to diffusional and kinetic parameters.

The insights for tentative identification of different molecules is based on the equations from Randles-Sevcik [21], which for irreversible electrochemical processes with one or multiple electrons can be applied according to equations 1 and 2, respectively: 


$$
\begin{aligned}
& I p=2.99 \times 10^{5} n \mathrm{AC}(\beta n \mathrm{D} v)^{1 / 2} \\
& I_{p}=2.99 \times 10^{5}\left(n^{\prime}+\beta_{n^{\prime}+1}\right)^{1 / 2} n D^{1 / 2} C_{o} A v^{1 / 2}
\end{aligned}
$$

Where, Ip is the peak current in A, n' is the sum of the number of electrons involved before the electron transfer slow step and $\beta n^{\prime}+1$ and the transfer coefficient of this rate determining, $\mathrm{n}$ is the total number of electrons involved, $\mathrm{D}$ is the diffusion coefficient of the electroactive specie in $\mathrm{cm} 2 \mathrm{~s}-1, \mathrm{~A}$ is the geometric area of working electrode in $\mathrm{cm} 2$,

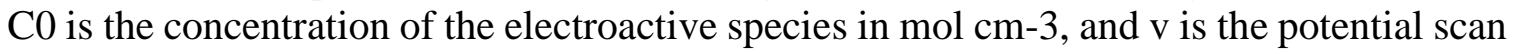
rate in $\mathrm{V} \mathrm{s}-1$.

As can been seen by this equation it is possible to keep constant all conditions and to change only the scan rate. In this context, first the slope for $I_{P} v S$ scan rate is verified for a standard of the analyte in the same conditions of the sample and after that a new study is made with this slope for pharmaceutical samples containing the respective analyte. In addition, as each electroactive molecule on working electrode can have different $D$ and $n$, and $\beta n '+1$, the slope of this curve should be different for each analyte and can be used for a possible identification in pharmaceutical samples such as have been shown in Table 2.

Furthermore, using the same equation above and keeping some constant conditions, as same working electrode and scan rate, the sensitivity of an electroactive molecule for electrochemical detection depend on of the same terms $D$ and $n$, and $\beta n^{\prime}+1$ of analyte. Thereby, the slope of calibration curve (Ip vs C) could also be used for a possible identification of an analyte (Table 2).

This evaluation is possible because all of them the value of $\mathrm{n}$ for their first oxidation processes have reported to be 2 [22-28]. In this context, using equation 2 for all studied molecules only two parameters D and B of these analytes can be used to explain the difference in slopes. In general, according to values of $\mathrm{D}$ that it have been reported for each molecules when D is higher for an analyte higher the slopes for calibration curve (sensitivity) and for scan rate study are. The D for each molecule is related with its size and charge as well as with used medium (electrolyte and $\mathrm{pH}$ ). On the other hand, the transfer coefficient of this rate determining $\left(\beta \mathrm{n}^{\prime}+1\right)$ needs also be considered. This term is related to an activation overpotential that each electroactive molecule has on a working electrode surface. This happens because each electroactive analyte has different redox groups and these can offer an electrochemical process easier or harder depending on used working electrode as well as of electrochemical conditions [24,26,27,31,32].

The electrochemical behavior with its respective proposed mechanism for the majority studied molecules has been reported and the number of involved electrons and protons as well as the electroactive group for each molecule can be seen in new table added in the supplementary information (Scheme S1). In these new references added can be seen the suggestion of the redox mechanism for each studied molecule, but using different working electrodes. 
Thus, it can be inferred that both slope approaches (Figure 4A and B) provide suitable selectivity to allow the direct identification of raw material samples that presents distinguishable redox behavior (Scheme, S1), as well as, of drugs present in dosage forms, that are featured as matrices of low complexity (Table 2).

TABLE 2. Slopes obtained from $I_{\mathrm{pa}} v s . v^{1 / 2}$ plot and calibration graph.

\begin{tabular}{lccccc}
\hline \multirow{2}{*}{ Drug } & $E_{\mathrm{p} 1 \mathrm{a} / 2 \mathrm{a}}$ & \multicolumn{2}{c}{$I_{\mathrm{pa}} v s . v^{1 / 2}$ slopes** $^{*}$} & \multicolumn{2}{c}{ Calibration curve slopes* } \\
\cline { 3 - 6 } & & $\mathrm{S}$ & $\mathrm{DF}$ & $\mathrm{S}$ & $\mathrm{DF}$ \\
\hline PAR & 0.35 & $0.39 \pm 2.2$ & $0.39 \pm 2.4$ & $0.19 \pm 2.1$ & $0.19 \pm 3.1$ \\
PMZ & $0.52 / 0.75$ & $1.25 \pm 3.7$ & $1.25 \pm 5.1$ & $0.10 \pm 2.8$ & $0.10 \pm 4.0$ \\
DIC & 0.55 & $0.24 \pm 4.9$ & $0.24 \pm 5.2$ & $0.06 \pm 5.5$ & $0.06 \pm 3.9$ \\
IND & 0.75 & $1.24 \pm 4.9$ & $1.24 \pm 5.1$ & $0.002 \pm 4.2$ & $0.002 \pm 5.3$ \\
PRX & 0.55 & $0.40 \pm 3.3$ & $0.40 \pm 3.4$ & $0.02 \pm 6.1$ & $0.02 \pm 4.6$ \\
CBP & $0.65 / 0.90$ & $0.77 \pm 7.7$ & $0.77 \pm 8.3$ & $0.03 \pm 6.0$ & $0.03 \pm 4.8$ \\
\hline
\end{tabular}

Legend: S (standard) and DF (dosage form) assay solutions.

$* \mu \mathrm{A}(\mathrm{mmol} . \mathrm{L})^{-1} ; * *\left(\mathrm{mV} . \mathrm{s}^{-1}\right)^{1 / 2}, \mathrm{RSD}$ values $<8 \%(\mathrm{n}=3)$.

In order to validate the difference between the slopes of each drug calculated with the calibration curves and to confirm that these parameters do not deviate between standard and dosage forms, statistical analysis ANOVA and Tukey's test were performed, with 0.05 significance level $(\alpha=0.05)$ (Figure 6).

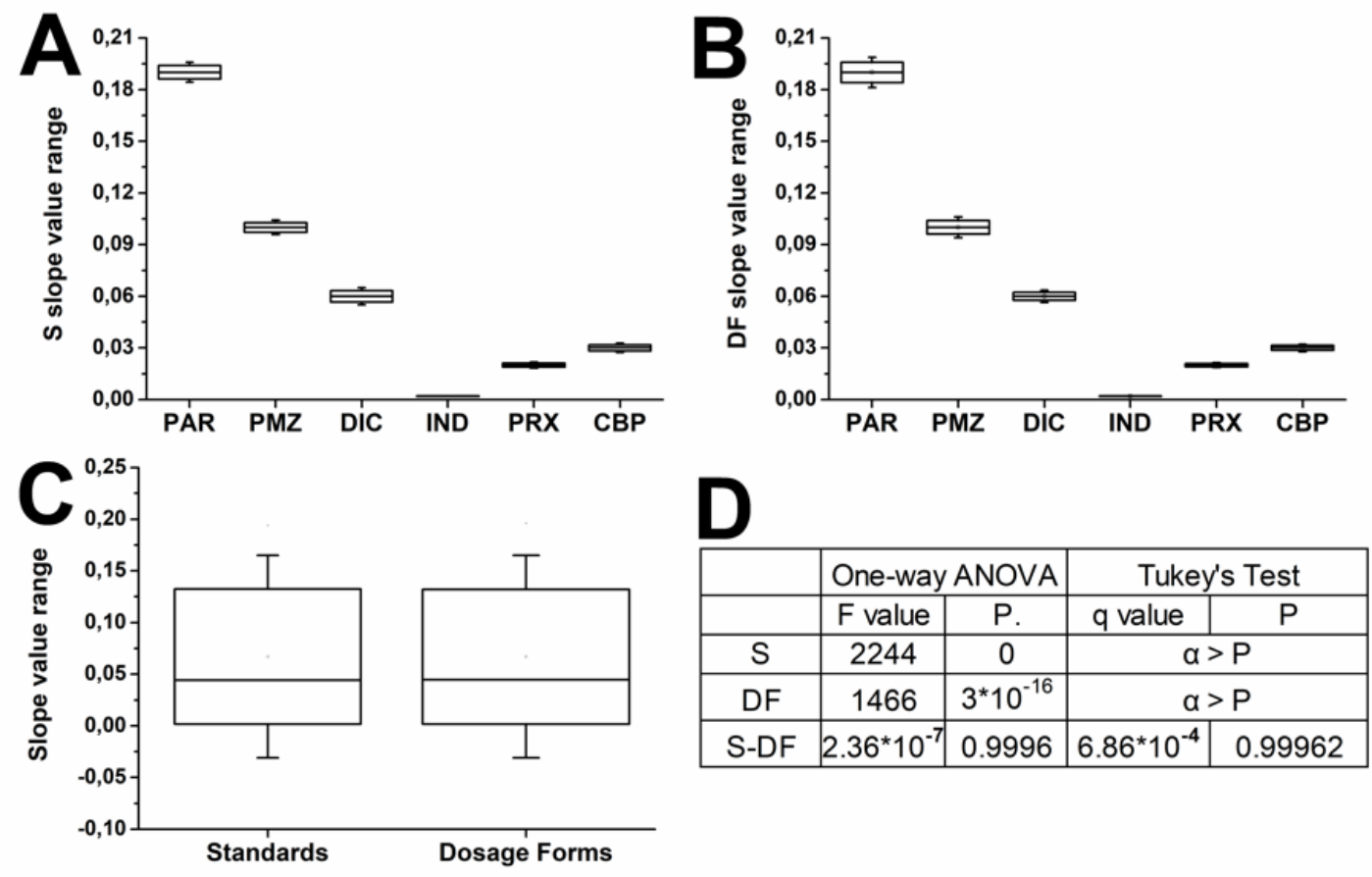

Figure 6. Graphical box charts of slope value ranges of the standards (A), dosage forms (B) calculated with the calibration graphs, between both of them (C) and statistical analysis of A: standard, B:dosage form and C:standard-dosage form datasets (S, DF and S-DF respectively) with ANOVA and Tukey's test (D) (Null Hypothesis: The means of all levels are equal; Alternative Hypothesis: The means of one or more levels are different). 
The calculated P-values for the slope values of the standards (Figure 6) and dosage forms (Figure 6B) were smaller than the significance level (Figure 6D) ( $\alpha>$ P), thus, each slope value for each individual drug is sufficiently different to be regarded as a unique parameter for identification. The Tukey's test was performed for each drug combination (PAR, PMZ, DIC, IND, PRX and CBP) in pairs. The P-value was also smaller than the significance level $(\alpha>\mathrm{P})$, thus, indicating that the difference of the slopes are significant between each paired drug. In the ANOVA analysis of the dataset created to compare standards and dosage forms (Figure 6C), as expected, the null hypothesis was validated and the same conclusion was achieved in the Tukey's test $(\alpha<\mathrm{P})$. This indicates that the slopes between standard and dosage form do not differ and are both equally viable to be used for the identification of the drugs investigated in this study.

The presence of other electroactive species in samples can disturb the slopes and this is a limitation of the proposed method when a pharmaceutical formulation presents more than one drug. However, in this case, a simultaneous analysis of these drugs can be investigated for each specific pharmaceutical sample and if the potential peak for each analyte present a good resolution, it can also be possible to use slopes from $I_{\mathrm{p}} v s$ scan rate or $I_{\mathrm{p}} v S \mathrm{C}$ for a possible identification. It worth also mention that the changing in the slopes provide by presence of other electroactive species in the pharmaceutical sample could be used to identify a possible interferent in this sample.

The presence of other electroactive species in samples can disturb the slopes and this is a limitation of the proposed method when a pharmaceutical formulation presents more than one drug. However, in this case, a simultaneous analysis of these drugs can be investigated for each specific pharmaceutical sample and if the potential peak for each analyte present a good resolution, it can also be possible to use slopes from $I_{\mathrm{p}} v s$ scan rate or $I_{\mathrm{p}} v s \mathrm{C}$ for a possible identification. It worth also mention that the changing in the slopes provide by presence of other electroactive species in the pharmaceutical sample could be used to identify a possible interferent in this sample.

Finally, the reproducibility of the use of slope approach was tested in different days, with preparation of new solutions obeying all the standards of analysis being found RSD values approximate $8 \%$. In fact, different carbon electrodes would present different electrode kinetics. In fact, the slopes obtained at CPE presented differences visual and statically significant of PGE (Figure 5C, 5D, 5E). Nevertheless, any electrode materials presenting the same electroactive surface area and chemical properties can be applied.

Finally, owing to the use of PGE to quantify the drug samples, the recoveries ranged from 97 to $1035 \%$, whereas the RSD was lower than 5\% $(n=3)$. The linear regressions of all target samples are presented in Table 3.

TABLE 3. Analytical parameters of quantitative assay

\begin{tabular}{llcccc}
\hline Drug & $\begin{array}{l}\text { Linear Regression } \\
\text { Equation (Standards) }\end{array}$ & $\begin{array}{c}\text { Linear } \\
\text { correlation }(\mathrm{R})\end{array}$ & $\begin{array}{c}\text { Linear range } \\
\left(\mu \mathrm{mol.L} \mathrm{L}^{-1}\right)\end{array}$ & $\begin{array}{c}\text { LOD } \\
\left(\mu \mathrm{mol.L} \mathrm{L}^{-1}\right)\end{array}$ & $\begin{array}{c}\text { Recovery } \\
(\%)^{*}\end{array}$ \\
\hline PAR & $\mathrm{y}=-4.4 .10^{-7}+0.19 \mathrm{x}$ & 0.997 & $4.9-38$ & 2.50 & 97.6 \\
PMZ & $\mathrm{y}=-2.3 .10^{-7}+0.10 \mathrm{x}$ & 0.986 & $4.9-38$ & 4.40 & 97.1 \\
DIC & $\mathrm{y}=-2.3 .10^{-7}+0.06 \mathrm{x}$ & 0.985 & $9.9-56$ & 6.72 & 98.0 \\
IND & $\mathrm{y}=-7.5 .10^{-9}+0.002 \mathrm{x}$ & 0.996 & $15-107$ & 18.35 & 98.4
\end{tabular}




\begin{tabular}{llcccc} 
PRX & $y=1.1 .10^{-6}+0.02 x$ & 0.994 & $2.9-160$ & 39.81 & 103.6 \\
CBP & $y=-2.1 .10^{-7}+0.03 x$ & 0.991 & $18-60$ & 9.5 & 98.1 \\
\hline
\end{tabular}

*related to the labeled value

Thus, tilt profiles were statistically similar when compared to results from the same drug, with significant matching between patterns and samples. When comparing drugs different from each other, the slopes did not match. These results show that such a parameter may be a complementary tool for the identification of drugs in samples. These results are possibly due to the unique characteristics of the molecules that interfere both in the reaction kinetics and in the diffusional profile in the evaluated medium. Electrodes with distinct characteristics change the kinetic profile of the reactions.

Furthermore, the identification process in quality control must be assessed by three or more simple methods of low level of confidence, the voltammetric approach upsurges as a potential alternative (Table 2, supplementary material).

\section{Conclusions}

The methodology for compounds identification by using slope of the calibration curve (peak currents vs different concentrations) obtained by DPV, and the slope of the study of the speed of sweep (slope of square root of the speed and current, served as complementary tool to identify drugs. The low cost PGEs showed to be useful for quantitative and qualitative analysis of drug samples. The DPV profile for the target samples, herein investigated showed even more distinguishable features than the conventional Raman spectra, thus the proposed methodology is very promising in order to accurately identify pharmaceutical compounds in commercial samples.

\section{Acknowledgements}

The authors wish to thank Conselho Nacional de Desenvolvimento Científico e Tecnológico (CNPq) [grant number 471109/2013-4] and Financiadora de Estudos e Pesquisas (FINEP) [grant number 01.14.0140.00] for the financial support to this work. Coordenadoria de Aperfeiçoamento de Pessoal (CAPES) for Emily Moreno scholarships. CF would like to express his gratitude to PALS at RGU for its support

\section{References}

1. S. Görög, Drug safety, drug quality, drug analysis, J. Pharmaceut. Biomed. 48 (2008).

2. Y. Ishida, T. Fujita, K. Asai, New detection and separation method for amino acids by high-performance liquid chromatography, J. Chromatogr. A 204 (1981).

3. I. Y. L. Macêdo, E.S. Gil, Pencil and Paper Electrodes for Pharmaceutical Analyses, J. Anal. Pharm. Res. 4 (2017). 
4. G.A. Saleh, H.F. Askal, I.H. Refaat, A.H. Naggar, F.A.M. Abdel-aal, Adsorptive square wave voltammetric determination of the antiviral drug valacyclovir on a novel sensor of copper microparticles-modified pencil graphite electrode, Arab. J. Chem. 9 (2016).

5. E. Dede, Ö. Sağlam, Y. Dilgin, Sensitive voltammetric determination of niclosamide at a disposable pencil graphite electrode, Electrochim. Acta. 127 (2014).

6. I.Y.L. de Macedo, L.F. Garcia, A.R. de Souza, A.M.L. da Silva, C. Fernandez, M.D.G. Santos, R.S. Magalhães, I.M.S. Torres, E. de S. Gil, Differential pulse voltammetric determination of albendazole and mebendazole in pharmaceutical formulations based on modified sonogel carbon paste electrodes with perovskiteType $\mathrm{LaFeO}_{3} \mathrm{Nanoparticles,} \mathrm{J.} \mathrm{Electrochem.} \mathrm{Soc.} 163$ (2016).

7. M. Hasanzadeh, N. Shadjou, L. Saghatforoush, J.E.N. Dolatabadi, Preparation of a new electrochemical sensor based on iron (III) complexes modified carbon paste electrode for simultaneous determination of mefenamic acid and indomethacin, Colloid. Surface. 92 (2012).

8. A.R. Khaskheli, J. Fischer, J. Barek, V. Vyskočil, Sirajuddin and M.I. Bhanger, Differential pulse voltammetric determination of paracetamol in tablet and urine samples at a micro-crystalline natural graphite - polystyrene composite film modified electrode, Electrochim. Acta 101 (2013).

9. K. M. AlAqad, R. Suleiman, O.C.S. Al Hamouz, T.A. Saleh, Novel graphene modified carbon-paste electrode for promazine detection by square wave voltammetry, J. Mol. Liq. 252 (2018).

10. D. G. Dilgin and S. Karakaya, Differential pulse voltammetric determination of acyclovir in pharmaceutical preparations using a pencil graphite electrode, Mater. Sci. Eng. C. 63 (2016).

11. Y. Uchida, E. Kätelhön, R.G. Compton, Linear sweep voltammetry with nontriangular waveforms: New opportunities in electroanalysis, J. Electroanal. Chem. 818 (2018).

12. R.S. Das, Y.K. Agrawal, Raman spectroscopy: recent advancements, techniques and applications, Vib. Spectrosc. 57 (2011).

13. T. Vankeirsbilck, A. Vercauteren, W. Baeyens, G. Van der Weken, F. Verpoort, G. Vergote, J. Remon, Applications of Raman spectroscopy in pharmaceutical analysis, TrAC-Trend. Anal. Chem. 21 (2002).

14. O. Y. Rodionova, A. V. Titova, N. A. Demkin, K. S. Balyklova, A. L. Pomerantsev, Qualitative and quantitative analysis of counterfeit fluconazole capsules: A noninvasive approach using NIR spectroscopy and chemometrics, Talanta, 195 (2019).

15. N. L. Calvo, R. M. Maggio, T. S. Kaufman, Characterization of pharmaceutically relevant materials at the solid state employing chemometrics methods, J. Pharm.Biomed. Anal. 147 (2018).

16. E. O. Barnes, X. Chen, P.Li, R.G. Compton, Voltammetry at porous electrodes: A theoretical study, J. Electroanal. Chem. (2014).

17. J. Hao, L. Ji, K. Wu, N. Yang, Electrochemistry of ZnO@reduced graphene oxides, Carbon 130 (2018).

18. N. Kumar, A. Bansal, G. S. Sarma, R. K. Rawal, Chemometrics tools used in analytical chemistry: An overview. Talanta, 123 (2014).

19. Y. Roggo, K. Degardina, P. Margot, Identification of pharmaceutical tablets by Raman spectroscopy and chemometrics, Talanta, 81 (2010). 
20. B. L. Milman, Identification of chemical compounds, TrAC-Trend. Anal. Chem. 24 (2005) 493-508. doi.org/10.1016/j.trac.2005.03.013.

21. C. R. Guy, B. Craig E. Understanding Voltammetry. World Scientific Publishing Company (2nd Edition) (2011).

22. A. C. F. Ribeiro, M. C.F. Barros, L. M. P. Veríssimo, C. I. A. V. Santos, A. M. T. D. P. V. Cabral, G. D. Gaspar, M. A. Esteso. Diffusion coefficients of paracetamol in aqueous solutions. Chem. Thermodynamics, 97-99, 54 (2012).

23. P. Fanjul-Bolado, P. J. Lamas-Ardisana, D. Hernández-Santos, A. Costa-García. Electrochemical study and flow injection analysis of paracetamol in pharmaceutical formulations based on screen-printed electrodes and carbon nanotubes. Analytica Chimica Acta, 133-138, 638 (2009).

24. E. Honarmand, M. H. Motaghedifard, M. Hadi, H. Mostaanzadeh. Electrooxidation study of promethazine hydrochloride at the surface of modified gold electrode using molecular self assembly of a novel bis-thio Schiff base from ethanol media. Journal of Molecular Liquids, 429-439, 216 (2016).

25. M.M. Cid-Ceron, D.S. Guzman-Hernandez, M.T. Ramirez-Silva, A. G. M. Romero-Romo, M. Palomar-Pardave. Accepted Manuscript Title: New insigths on the kinetics and mechanism of the electrochemical oxidation of diclofenac in neutral aqueous medium. Electrochimica Acta, DOI: http://dx.doi.org/doi: 10.1016/j.electacta.2016.03.094. 2016.

26. R. N. Goyal, S. Chatterjee, B. Agrawal. Electrochemical investigations of diclofenac at edge plane pyrolytic graphite electrode and its determination in human urine. Sensors and Actuators B, 743-748, 145 (2010).

27. M. Arvand, T. M. Gholizadeh. Gold nanorods-graphene oxide nanocomposite incorporated carbon nanotube paste modified glassy carbon electrode for voltammetric determination of indomethacin. Sensors and Actuators B: Chemical. 622-632, 186 (2013).

28. S. Tahir, K. Yasmeen, M. Hanif, O. Khaliq, H. Muhammad, Hafsa, I. A. Tahiri, S. Jahangir, S. T. Ali. Electrochemical Methodology for NSAID's Determination and its Interaction with Steroid Dexamethasone. Int. J. Electrochem. Sci., doi: 10.20964/2019.06.16., 5748 - 5762, 14 (2019).

29. Kianoush Sarhangzadeh, Ali Akbar Khatami, Mohammad Jabbari, Siavash Bahari. Simultaneous determination of diclofenac and indomethacin using a sensitive electrochemical sensor based on multiwalled carbon nanotube and ionic liquid nanocomposite. DOI 10.1007/s10800-013-0609-3. J Appl Electrochem. 2013.

30. D. Nematollahi, H. Shayani-Jama, M. Alimoradi, S. Niroomand. Electrochemical oxidation of acetaminophen in aqueous solutions: Kinetic evaluation of hydrolysis, hydroxylation and dimerization processes. Electrochimica Acta, 7407-7415, 54 (2009).

31. A. Abbaspour, R. Mirzajani. Electrochemical monitoring of piroxicam in different pharmaceutical forms with multi-walled carbon nanotubes paste electrode. Journal of Pharmaceutical and Biomedical Analysis, 41-48, 44 (2007). 